\title{
The sudden presentation and progression of overt cervical metastases following treatment of head and neck cancers
}

\author{
Julia A. Woolgar • Alfio Ferlito • Robert P. Takes • \\ Juan P. Rodrigo $\cdot$ Carl E. Silver $\cdot$ Kenneth O. Devaney \\ Alessandra Rinaldo
}

Received: 26 October 2010/Accepted: 2 November 2010/Published online: 28 November 2010

(C) The Author(s) 2010. This article is published with open access at Springerlink.com

\section{Introduction}

In a small number of head and neck cancer patients, "explosive" growth of nodal metastasis occurs in the untreated neck following removal of the primary malignancy [1]. The neck-either ipsilateral and/or contralateral-appears free of metastasis on routine preoperative work-up including radiological assessment by an experienced, dedicated head and neck radiologist. Within a few weeks following surgery, nodal disease becomes overt and surprisingly advanced in terms of volume and extracapsular spread. Although well recognized by surgeons, radiotherapists, oncologists and pathologists, the prevalence and incidence rates of sudden metastatic presentation are unknown since the condition is yet to be clearly defined and explained. In the absence of residual or recurrent

This paper was written by members of the International Head and Neck Scientific Group (www.IHNSG.com).

J. A. Woolgar

Oral Pathology, University of Liverpool Dental Hospital,

Liverpool, UK

A. Ferlito $(\bowtie) \cdot$ A. Rinaldo

Department of Surgical Sciences, ENT Clinic,

University of Udine, Azienda Ospedaliero-Universitaria,

Piazzale S. Maria della Misericordia, I-33100 Udine, Italy

e-mail: a.ferlito@uniud.it

R. P. Takes

Department of Otolaryngology-Head and Neck Surgery, Radboud University Nijmegen Medical Center,

Nijmegen, The Netherlands

J. P. Rodrigo

Department of Otolaryngology,

Hospital Universitario Central de Asturias, Oviedo, Spain disease at the primary tumor site, it seems likely that the source of the rapidly growing nodal tumor is small metastases or micrometastases $(2 \mathrm{~mm}$ or less in profile diameter) [2-4] present within cervical lymph nodes, but undetected at the time of initial surgery. An alternative source could be tumor emboli released into the lymphovascular system during manipulation and surgical removal of the primary tumor and subsequent settling in a lymph node. This has been demonstrated in animal studies but its role in humans is uncertain [5].

In cases where disease is already present within the lymph node, it may be that the primary tumor was "dominant", preventing or suppressing growth of the nodal deposit(s) and that the rapid growth follows loss of the primary tumor's inhibitory influence. Although a number of processes may play a role in thwarting the expansion of microscopic tumors, one critical mechanism behind tumor dormancy is the ability of the tumor population to induce angiogenesis [6]. It is thought that angiogenesis plays a role

\author{
J. P. Rodrigo \\ Instituto Universitario de Oncología del Principado de Asturias, \\ Oviedo, Spain

\section{E. Silver} \\ Departments of Surgery and Otolaryngology-Head and Neck \\ Surgery, Albert Einstein College of Medicine, \\ Montefiore Medical Center, Bronx, NY, USA \\ K. O. Devaney \\ Department of Pathology, Allegiance Health, \\ Jackson, MI, USA
}


in the behavior of micrometastases and there is experimental evidence to suggest that removal of the primary tumor can promote the development of nodal disease by enhancing angiogenesis. In addition, it is possible that depression of the immune surveillance and immune systems, related to the trauma of surgery, may allow dormant or slow-growing nodal disease to flourish. On the other hand, while evidence for the role of psychosocial factors in cancer initiation is limited and equivocal, evidence is stronger for links between psychological factors such as stress, depression, and social isolation and disease progression. Stress-related processes (such as the treatment of the primary tumor) can impact pathways implicated in cancer progression, including immunoregulation, angiogenesis, and invasion. Contributions of systemic factors, such as stress hormones, to the crosstalk between tumor and stromal cells, appear to be critical in modulating downstream signaling pathways with important implications for disease progression [7].

The status of isolated tumor cells (ITC) and their relationship to micrometastases remains uncertain. Staining for the proliferation marker Ki-67 shows positive cells in $29 \%$ of ITCs, $92 \%$ of micrometastases and $96 \%$ of macrometastases [8]. However, the TNM classification states ITCs do not typically show evidence of proliferation or stromal reaction (indicators of metastatic activity), or penetration of vascular or lymphatic sinus walls, and uses size as the defining criterion (with ITCs measuring less than $0.2 \mathrm{~mm}$ and micrometastases $0.2-2 \mathrm{~mm}$ in greatest dimension) [4]. Unlike conventional metastases, ITCs do not have a specific blood supply and rely on passive diffusion for oxygen and nutrient supply $[9,10]$. It is thought that the lack of a specific blood supply limits the growth of the tumor deposits and cytokinetic and apoptotic rates may be matched for long periods so that an equilibrium exists (tumor dormancy) until they are detected and eradicated by immune surveillance, or acquire a specific blood supply that allows tumor growth [11]. The prevalence of dormant tumor cells is much greater than previously thought. Both clinical and experimental data suggest that dissemination can occur at very early stages of tumor growth and that disseminated cells can remain dormant rather than grow progressively. Such asymptomatic dormancy of disseminated cells can persist for decades. However, little is known about the mechanisms responsible for the regulation of dormancy in these cells [6]. Several mechanisms have been proposed to explain the onset of tumor dormancy and the nature of the shift responsible for releasing these dormant tumor cells from growth restraints. One such mechanism is the induction of angiogenesis. According to this approach, the escape of tumors from dormancy depends on the tumor population undergoing an angiogenic switch that induces the recruitment and formation of new and functional tumor vasculature [12]. Hypoxia and inflammatory mediators are powerful stimuli for angiogenesis $[13,14]$. Vascular endothelial growth factor (VEGF) and the fibroblast growth factor (FGF) family are two of the more widely researched molecules in the angiogenic cascade (for review, see Zimmerman et al. [13]). VEGF overexpression in breast [15], gastrointestinal [16], and colorectal [17] carcinomas is associated with the metastatic phenotype and is predictive of a poor prognosis. Moreover, it seems likely that angiogenic factors released in response to wounding at the primary tumor site may enhance the growth of micrometastases and activate dormant nodal deposits [18-20].

In addition, it is possible that some primary tumors produce angiostatin that inhibits angiogenesis [10]; hence, loss of the suppressive effect following surgery on the primary tumor removes the suppressive effect and promotes metastatic growth [21-23]. The precise mechanisms are not fully understood and it seems that some angiogenesis inhibitors control metastatic growth by inducing apoptosis in tumor cells [11, 24].

Although molecular and cellular components of the "angiogenic switch" play central roles in the transition of non-angiogenic tumors from the dormancy stage to rapid tumor mass expansion, many other unrelated factors can play major roles in tumor dormancy. Tumor dormancy in some cancers can be induced by potent immunosuppression of tumor growth by the immune system, where equilibrium between the immune system and the tumor cells results in long-term persistence of tumor dormancy. There is accumulating evidence that cellular as well as humoral responses are required for immune surveillance and the maintenance of tumor dormancy $[6,25]$. The immune system, however, appears to both prevent and promote cancer. Indeed, various bone marrow derived cells and inflammatory cells had been implicated in promoting tumor progression and development by inducing inflammation and angiogenesis [26]. The inflammatory process subsequent to surgical resection of the primary tumor could promote the growth of dormant micrometastases and ITCs.

Indeed, the inflammatory process associated with surgery shares a number of central mediators and pathways with tumor growth and invasiveness. Both cellular components (mainly macrophages and fibroblasts) and humoral factors associated with inflammation have been shown to enhance tumor growth in numerous preclinical studies. These studies have shown that removal of the primary cancer reactivates proliferative and metastatic pathways in residual tumor. Relapse of disease has therefore been related to (surgical) trauma and consequent inflammation [20, 27].

However, the role of the immune system in nodal metastasis in head and head malignancies is also poorly 
understood. In malignant melanoma, tumor-reactive cytotoxic T-lymphocytes are present in the tumor and the blood [28] and spontaneous regression of the primary or metastatic tumor is well recognized [29]. It has been suggested that the primary melanoma might function as a "booster vaccine" constantly providing tumor antigens keeping the immune system alerted [29]. Eventually, in most patients, the tumor will escape the immune system although this may follow a prolonged period of dormancy. The phenomenon of sudden, explosive growth of nodal metastases following removal of the primary melanoma is recognized but is considered unusual and whether it is due to changes in systemic or local immunity or angiogenesis or other as yet undefined mechanisms is uncertain [29].

As in malignant melanoma, the explosive growth of nodal deposits following removal of the primary tumor in head and neck cancers is unusual and contrasts sharply with the more usual presentation of relapse in the nontreated ipsilateral or contralateral neck when disease presents later as a slow-growing deposit often localized to a single node [30].

Other factors have also been described in relation to activation of dormant tumor cells. For example, in the in vitro studies, induction of fibrosis, associated with deposition of type I collagen in a metastatic microenvironment, was demonstrated to induce dormant cells to form proliferative metastatic lesions through beta1-integrin signaling [31].

Although the discussed mechanisms may explain, at least in part, dormancy of undetected metastatic cells, the "explosive" speed of the growth of nodal metastasis after removal of the primary tumor still remains an intriguing phenomenon.

Open Access This article is distributed under the terms of the Creative Commons Attribution Noncommercial License which permits any noncommercial use, distribution, and reproduction in any medium, provided the original author(s) and source are credited.

\section{References}

1. Woolgar JA, Triantafyllou A (2010) Lymph node metastases in head and neck malignancies: assessment in practice and prognostic importance. Diagn Histopathol (Oxf) 16:265-275

2. Devaney KO, Rinaldo A, Ferlito A (2007) Micrometastases in cervical lymph nodes from patients with squamous carcinoma of the head and neck: should they be actively sought? Maybe. Am J Otolaryngol 28:271-274

3. Ferlito A, Rinaldo A, Devaney KO, Nakashiro K, Hamakawa H (2008) Detection of lymph node micrometastases in patients with squamous carcinoma of the head and neck. Eur Arch Otorhinolaryngol 265:1147-1153

4. Sobin LH, Gospodarowicz MK, Wittekind Ch (2009) UICC TNM classification of malignant tumours, 7th edn. Wiley-Blackwell, New York
5. Nishizaki T, Matsumata T, Kanematsu T, Yasunaga C, Sugimachi K (1990) Surgical manipulation of VX2 carcinoma in the rabbit liver evokes enhancement of metastasis. J Surg Res 49:92-97

6. Almog N (2010) Molecular mechanisms underlying tumor dormancy. Cancer Lett 294:139-146

7. Lutgendorf SK, Sood AK, Antoni MH (2010) Host factors and cancer progression: biobehavioral signaling pathways and interventions. J Clin Oncol 28:4094-4099

8. Yanagita S, Natsugoe S, Uenosono Y, Kozono T, Ehi K, Arigami $\mathrm{T}$, Arima H, Ishigami S, Aikou T (2008) Sentinel node micrometastases have high proliferative potential in gastric cancer. J Surg Res 145:238-243

9. Atula T, Hunter KD, Cooper LA, Shoaib T, Ross GL, Soutar DS (2009) Micrometastases and isolated tumour cells in sentinel lymph nodes in oral and oropharyngeal squamous cell carcinoma. Eur J Surg Oncol 35:532-538

10. Seethela RR (2009) Current state of neck dissection in the United States. Head Neck Pathol 3:238-245

11. Holmgren L, O'Reilly MS, Folkman J (1995) Dormancy of micrometastases: balanced proliferation and apoptosis in the presence of angiogenesis suppression. Nat Med 1:149-153

12. Aguirre-Ghiso JA (2007) Models, mechanisms and clinical evidence for cancer dormancy. Nat Rev Cancer 7:834-846

13. Zimmerman MA, Selzman CH, Harken AH (1999) Surgical implications of therapeutic angiogenesis. Surgery 125:243-249

14. Petruzzelli GJ (1996) Tumor angiogenesis. Head Neck 18:283-291

15. Yoshiji H, Gomez DE, Shibuya M, Thorgeirsson UP (1996) Expression of vascular endothelial growth factor, its receptor, and other angiogenic factors in human breast cancer. Cancer Res 56:2013-2016

16. Brown LF, Berse B, Jackman RW, Tognazzi K, Manseau EJ, Senger DR, Dvorak HF (1993) Expression of vascular permeability factor (vascular endothelial growth factor) and its receptors in adenocarcinomas of the gastrointestinal tract. Cancer Res 53:4727-4735

17. Nakata S, Ito K, Fujimori M, Shingu K, Kajikawa S, Adachi W, Matsuyama I, Tsuchiya S, Kuwano M, Amano J (1998) Involvement of vascular endothelial growth factor and urokinasetype plasminogen activator receptor in microvessel invasion in human colorectal cancers. Int J Cancer 79:179-186

18. O'Reilly MS, Boehm T, Shing Y, Fukai N, Vasios G, Lane WS, Flynn E, Birkhead JR, Olsen BR, Folkman J (1997) Endostatin: an endogenous inhibitor of angiogenesis and tumor growth. Cell 88:277-285

19. Cao Y, O'Reilly MS, Marshall B, Flynn E, Ji RW, Folkman J (1998) Expression of angiostatin cDNA in a murine fibrosarcoma suppresses primary tumor growth and produces long-term dormancy of metastases. J Clin Invest 101:1055-1063

20. Maniwa Y, Okada M, Ishii N, Kiyooka K (1998) Vascular endothelial growth factor increased by pulmonary surgery accelerates the growth of micrometastases in metastatic lung cancer. Chest 114:1668-1675

21. Soff GA (2000) Angiostatin and angiostatin-related proteins. Cancer Metastasis Rev 19:97-107

22. Dell'Eva R, Pfeffer U, Indraccolo S, Albini A, Noonan D (2002) Inhibition of tumor angiogenesis by angiostatin: from recombinant protein to gene therapy. Endothelium 9:3-10

23. Matsumoto G, Ohmi Y, Shindo J (2001) Angiostatin gene therapy inhibits the growth of murine squamous cell carcinoma in vivo. Oral Oncol 37:369-378

24. Luzzi KJ, MacDonald IC, Schmidt EE, Kerkvliet N, Morris VL, Chambers AF, Groom AC (1998) Multistep nature of metastatic inefficiency: dormancy of solitary cells after successful extravasation and limited survival of early micrometastases. Am J Pathol 153:865-873 
25. Uhr JW, Scheuermann RH, Street NE, Vitetta ES (1997) Cancer dormancy: opportunities for new therapeutic approaches. Nat Med 3:505-509

26. Shaked Y, Voest EE (2009) Bone marrow derived cells in tumor angiogenesis and growth: are they the good, the bad or the evil? Biochim Biophys Acta 1796:1-4

27. Ceelen WP, Morris S, Paraskeva P, Pattyn P (2007) Surgical trauma, minimal residual disease and locoregional cancer recurrence. Cancer Treat Res 134:51-69

28. Robbins PF, Kawakami Y (1996) Human tumor antigens recognized by T cells. Curr Opin Immunol 8:628-636
29. De Giorgi V, Massi D, Gerlini G, Mannone F, Quercioli E, Carli $P$ (2003) Immediate local and regional recurrence after the excision of a polypoid melanoma: tumor dormancy or tumor activation? Dermatol Surg 29:664-667

30. Woolgar JA (2006) Salvage neck dissections in oral and oropharyngeal squamous cell carcinoma: histological features in relation to disease category. Int J Oral Maxillofac Surg 35:907-912

31. Barkan D, El Touny LH, Michalowski AM, Smith JA, Chu I, Davis AS, Webster JD, Hoover S, Simpson RM, Gauldie J, Green JE (2010) Metastatic growth from dormant cells induced by a colI-enriched fibrotic environment. Cancer Res 70:5706-5716 